\title{
A prática clínica em fitoterapia magistral: uma experiência interprofissional da Associação Brasileira de Fitoterapia
}

\author{
Maria Angélica Fiut ${ }^{\mathrm{a},}$, Gabriela Deutsch ${ }^{\mathrm{a}}$, Liziene Arruda ${ }^{\mathrm{a}}$, Deborah Marques ${ }^{\mathrm{a}}$, Paulo \\ Henrique Leda ${ }^{\mathrm{a}, \mathrm{b}}$ Alex Botsaris ${ }^{\mathrm{a}}$, Antonio Carlos Seixlack ${ }^{\mathrm{a}}$ \\ ${ }^{\text {a }}$ Associação Brasileira de Fitoterapia (ABFIT), Rio de Janeiro, RJ, Brasil \\ ${ }^{\mathrm{b}}$ Escola Nacional de Saúde Pública ENSP/FIOCRUZ, Rio de Janeiro, RJ, Brasil
}

\section{Histórico do Artigo \\ Recebido em: $16 / 02 / 2018$ \\ Aceito em: \\ $30 / 03 / 2018$}

Palavras-chave: Práticas integrativas e complementares; fitoterapia; qualificação profissional.

Keyword: Integrative and complementary practices; phytotherapy; professional qualification.

\begin{abstract}
RESUMO
Este artigo tem o objetivo de relatar a experiência interprofissional da prática clínica ambulatorial em fitoterapia magistral, inserida no programa de qualificação profissional realizada pela Associação Brasileira de Fitoterapia (ABFIT) em um hospital público de grande porte na cidade do Rio de Janeiro. Trata-se de um estudo descritivo, com abordagem qualitativa que incorpora relatos de experiência e levantamentos bibliográficos para a produção de dados. A prática clínica ambulatorial aqui relatada tem como base teórica a combinação de elementos médicos tradicionais e conhecimentos técnico-científicos, em especial da Medicina Tradicional Chinesa. A integração desses fundamentos possibilita que a prescrição da fitoterapia seja executada por meio da associação de extratos vegetais em formulações magistrais que atendem à demanda individual do usuário. Essa abordagem permite ao aluno reunir indicações farmacológicas e tradicionais, proporcionando como resultado a maior eficácia do tratamento. Acredita-se que a experiência da ABFIT possa fornecer subsídios para o desenvolvimento de um modelo de atenção em saúde inovador no campo das Práticas Integrativas Complementares (PICs).
\end{abstract}

The clinical practice in master phytotherapy: an interprofessional experience of the Brazilian Association of Phytotherapy

\section{ABSTRACT}

This article aims to report the interprofessional experience of clinical practice in master phytotherapy which is part of the professional qualification program performed by the Brazilian Association of Phytotherapy (ABFIT) at public hospital in Rio de Janeiro city. It is a descriptive study with a qualitative approach that incorporates reports of experience and bibliographical surveys for the data production. The outpatient clinical practice reported here is based on the theoretical combination of traditional medical elements and technical-scientific knowledge, especially of Traditional Chinese Medicine. The integration of these fundamentals makes it possible to prescribe phytotherapy through the association of vegetal extracts in magisterial formulations that meet the individual demand of the user. This approach allows the student to gather pharmacological and traditional indications, resulting in greater effectiveness of the treatment. It is believed that ABFIT's experience can provide support for the development of an innovative health care model in the field of Complementary Integrative Practices (PICs).

\section{Introdução}

As plantas medicinais fazem parte da cultura popular há milhares de anos, porém, nas últimas décadas, o interesse pela fitoterapia tem crescido consideravelmente para usuários e pesquisadores (1). Essa prática ganha cada vez mais atenção da indústria e de diversos serviços de saúde (1). A Organização Mundial de Saúde (OMS), em 1978, reconheceu que $80 \%$ da população dos países em desenvolvimento utilizavam práticas tradicionais nos serviços de saúde e que, em $85 \%$, estavam presentes plantas ou preparações dessas. Desde então, a OMS tem se posicionado a respeito da necessidade

\footnotetext{
*Autor correspondente: angelicafiut@bol.com.br (M. A. Fiut)
} 
de valorização de plantas medicinais no âmbito da atenção primária à saúde $(2,3,4)$.

No Brasil, a temática foi levantada em diversas oportunidades como na $8^{\text {a }}$ Conferência Nacional de Saúde, em 1986 (5). Nessa ocasião, foi apresentada a experiência exitosa do Projeto Farmácias Vivas da Universidade Federal do Ceará em ações com o emprego de plantas medicinais nos serviços de atenção primária (5).

Desde então, alguns municípios brasileiros têm implantado o programa de fitoterapia nos serviços públicos de saúde como é o caso de Vitória (ES) (6), Curitiba (PR) (7), Rio de Janeiro (RJ) (8), Ribeirão Preto (SP) (9), Itapipoca (CE) (10), Juiz de Fora (MG) (11) e Campinas (SP) (12). Segundo o levantamento do Ministério da Saúde, em 2004, verificou-se que a fitoterapia esteve presente em 116 municípios brasileiros, contemplando 22 unidades federações (13). As experiências desses municípios revelaram uma importância na redução do custo, uma vez que possibilita maior prevenção de comorbidades, diminuindo possíveis internações hospitalares (6). Alguns questionamentos de ordem técnica também são relevantes, como o desempenho de iniciativas como o treinamento dos profissionais e a padronização das plantas medicinais de acordo com critérios técnico-científicos (6). Além disso, os relatos apontam para a necessidade de maior esclarecimento acerca da integração entre o saber popular e os resultados clínicos do uso de plantas medicinais no controle das doenças (6).

Com a instituição da Política Nacional de Práticas Integrativas e Complementares (PNPIC) no Sistema Único de Saúde (SUS), em 2006, foi possível reorganizar a implantação e implementação de ações e de serviços no SUS. Esta inclui a fitoterapia, com objetivo de assegurar a prevenção, promoção e recuperação da saúde na atenção primária. Propõe, ainda, o cuidado integral e humanizado, a fim de contribuir para o aumento da demanda de saúde com qualidade, eficácia, efetividade, segurança, controle, participação social e sustentabilidade (13).

Dentro desse contexto, a Associação Brasileira de Fitoterapia (ABFIT), fundada em 1998 como Instituto Brasileiro de Plantas Medicinais (IBPM), participou e contribuiu para o reconhecimento oficial da fitoterapia no Brasil, ao mesmo tempo que manteve o seu foco na qualificação de profissionais de saúde direcionada para a fitoterapia magistral. Seu principal objetivo é aliar tradição e ciência, a fim de contribuir para o alcance dos objetivos das práticas integrativas e complementares (PICs) (13). Seus cursos de qualificação profissional são ofertados com abordagem interprofissional, proporcionando ao aluno (profissional de saúde) o reconhecimento da necessidade do saber técnico-científico sobre a fitoterapia, e dos parâmetros tradicionais em relação às plantas medicinais com treinamento prático para aplicação no cuidado em saúde.

Portanto, o presente artigo tem o objetivo de relatar a experiência da prática clínica interprofissional no Ambulatório de Fitoterapia Magistral do Hospital Federal do Andaraí, a qual está inserida no programa de qualificação profissional como disciplina de prática clínica dos cursos da ABFIT.

\section{Materiais e Métodos}

Trata-se de um estudo de caso qualitativo de experiência pedagógica por metodologia teórico-prática da atividade clínica interprofissional do Ambulatório de Fitoterapia Magistral da ABFIT vinculada ao hospital público de grande porte na cidade do Rio de Janeiro. O estudo surgiu da iniciativa de compartilhar com o público leitor como se dá o projeto ambulatorial de fitoterapia magistral da ABFIT, que tem como construção a prática clínica interprofissional, bem como o uso de fitoterápicos magistrais na produção do cuidado em saúde. Além disso, este projeto faz parte do conteúdo programático de qualificação profissional dos cursos ofertados pela ABFIT. 
Dessa forma, julgou-se pertinente a descrição de três eixos temáticos:

\section{I - Projeto ambulatorial de fitoterapia magistral}

Em 2012, a ABFIT resgatou o projeto ambulatorial de fitoterapia iniciado no Posto de Saúde da Escola Nacional de Saúde Pública (ENSP/Fiocruz), onde ficou de 2005 a 2011. Este projeto é, atualmente, desenvolvido no hospital público de grande porte na cidade do Rio de Janeiro, com atendimentos que ocorrem no ambulatório de fitoterapia magistral, propiciando aos alunos a vivência da prática clínica numa perspectiva interprofissional. É importante ressaltar que o projeto conta com a parceria de farmácias magistrais privadas que manipulam e dispensam sem custos os fitoterápicos para os pacientes atendidos.

\section{II - Programa de qualificação profissional da ABFIT}

A fundamentação é trabalhada, anteriormente, nas aulas teóricas, quando os alunos adquirem conhecimento técnico-científico em fitoterapia e em elementos dos sistemas médicos tradicionais com grande ênfase na Medicina Tradicional Chinesa (MTC). Os atendimentos contam com preceptores das áreas de medicina, farmácia e nutrição, que acompanham o processo de ensino-aprendizagem do aluno, utilizando fitoterapia magistral no cuidado em saúde.

\section{III - Prescrições de fitoterápicos magistrais no ambulatório da ABFIT:}

Quanto ao acesso ao fitoterápico magistral, os usuários atendidos retornam no prazo de 7 a 10 dias após a última consulta para receberem gratuitamente suas formulações. A dispensação e orientação quanto ao uso correto dos fitoterápicos magistrais é realizada pelos farmacêuticos da equipe. Este projeto conta com a parceria de quatro farmácias magistrais licenciadas pelos órgãos competentes que manipulam as drogas vegetais preconizadas no memento de fitoterápicos elaborado pela ABFIT.

Para análise e discussão dos eixos temáticos foram utilizados artigos de periódicos, teses, dissertações, capítulos de livros, nacionais e internacionais, indexados em bases científicas.

Para a busca, foram consultadas as seguintes bases de dados brasileiras, uma vez que este projeto ocorre sobre a perspectiva do SUS: Scielo, Lilacs e Google Acadêmico. Na estratégia de busca, foram consultados os descritores: Fitoterapia; Plantas Medicinais; Práticas de Saúde Integrativas e Complementares; Medicina Alternativa; Medicina Complementar; Terapias Alternativas; Medicina Complementar e Integrativa; Práticas Integrativas e Complementares; Medicina Integrativa e Complementar; Práticas Complementares e Integrativas como descritores no período dos últimos 10 anos.

\section{Resultados e Discussão}

\section{O projeto ambulatorial de fitoterapia magistral}

O Ambulatório de Fitoterapia Magistral da ABFIT foi criado com o objetivo de qualificar o profissional de saúde para a prática clínica em fitoterapia a fim de assegurar melhor o cuidado em saúde da população. O ambulatório está instalado, desde 2012, no espaço físico do Setor de Clínica da Dor e Práticas Integrativas e Complementares do hospital público de grande porte na cidade do Rio de Janeiro. Atualmente, mantém-se como unidade de fitoterapia que atende pacientes encaminhados principalmente dos setores de Oncologia, Clínica da Dor e Cirurgia Plástica. Os atendimentos ocorrem em dois turnos semanais, totalizando 10 horas. 
A equipe do ambulatório é composta por profissionais/preceptores de medicina (1), nutrição (2), e farmácia (2), que participam coletivamente da consulta. A organização da interconsulta pode ser dividida em três etapas: a primeira pode ser realizada por médico, farmacêutico ou nutricionista, para coletar as informações pessoais do usuário, anamnese geral e específica, observação da língua e palpação do pulso, conforme a MTC. A segunda etapa consiste no recordatório alimentar de 24 horas e construção do plano alimentar pelo nutricionista. A última etapa refere-se à decisão conjunta do diagnóstico pela MTC, bem como a escolha dos fitoterápicos que melhor atendam a situação de saúde e queixa principal do usuário.

A lógica de trabalho é pautada na busca de construção interprofissional, segundo uma matriz transversal baseada no desenvolvimento de uma interlocução com a equipe de saúde da própria unidade, bem como os outros setores do hospital. O trabalho em equipe interprofissional é considerado a melhor forma de interação com os usuários devido à complexidade do cuidado em saúde, bem como a possibilidade de alcançar a eficiência na abordagem dos pacientes e nos resultados terapêuticos (14).

Ao longo da prática ambulatorial, a integralidade possibilita a construção compartilhada de respostas assistenciais e a otimização do cuidado em saúde (14). Assim, o aluno vivencia a colaboração interprofissional (14) num espaço comum, bem como o processo de comunicação e tomadas de decisões compartilhadas para a melhor produção do cuidado em saúde.

Outro parâmetro importante a ser observado é que o ambulatório de fitoterapia magistral está pautado na fundamentação da Medicina Tradicional Chinesa (MTC), caracterizada por um sistema médico integral, originado, há milhares de anos, na China $(13,15)$. Como fundamento, a MTC aponta a teoria do Yin-Yang, divisão do mundo em duas forças ou princípios fundamentais, interpretando os fenômenos em opostos complementares. O objetivo desse conhecimento é obter meios de equilibrar essa dualidade $(13,15)$.

Também inclui a teoria dos cinco movimentos, que atribui a todas as coisas e fenômenos, na natureza, assim como no corpo, uma das cinco energias (madeira, fogo, terra, metal, água) $(13,15)$. Utiliza como elementos a anamnese, palpação do pulso, observação da face e língua em suas várias modalidades de tratamento como: acupuntura, plantas medicinais, dietoterapia, práticas corporais e mentais $(13,15)$.

Para a construção desse memento, a ABFIT selecionou 47 espécies de drogas vegetais fundamentadas nos critérios preconizados pela diretriz da PNPIC (16), bem como informações baseadas na medicina em evidência e pesquisas científicas, com o intuito de assegurar a segurança e a eficácia da indicação terapêutica dos fitoterápicos (16). Tais espécies podem ser apresentadas nas prescrições como extrato seco padronizado, concentrado ou pó.

Algumas espécies de drogas vegetais (Arctostaphylos uva-ursi, Valeriana officinalis, Ginkgo biloba e Hypericum perforatum) que fazem parte do elenco de fitoterápicos do ambulatório da ABFIT, segundo as IN 02/2014 da ANVISA, estão restritas à prescrição médica; como o ambulatório conta com a equipe interprofissional, é possível trabalhar com tais plantas (16).

\section{Programa de qualificação profissional da ABFIT}

O programa da ABFIT possui, atualmente, as seguintes modalidades de qualificação profissional: educação a distância (EAD), curso de extensão presencial e Curso de PósGraduação Latu sensu de Fitoterapia na Prática Clínica recentemente aberto na plataforma da Instituição. 
A proposta de qualificação profissional da ABFIT é incentivar o aluno na participação da dinâmica do Ambulatório de Fitoterapia Magistral no hospital. Seu principal objetivo é propiciar a vivência na prática clínica numa perspectiva interprofissional mediante o uso de fitoterápicos magistrais na produção do cuidado em saúde.

O treinamento prático é a linha mestra que permite a reflexão e a elaboração conjunta do plano terapêutico para cada usuário do serviço. A participação do aluno nessa atividade permite ampliar sua compreensão sobre o papel de cada profissional e as interfaces necessárias à construção de alternativas às questões apresentadas pelo usuário. Além disso, também permite aplicar o saber técnico e científico de plantas medicinais e fitoterápicos.

A WHO (17) aponta que a educação interprofissional permite ao aluno aprender como interagir com os demais, não se superpondo ao currículo específico de cada área profissional, mas sendo complementar. $\mathrm{O}$ aprendizado se dá no contato direto com os usuários, tendo como objetivo comum responder às questões de saúde da população atendida.

De acordo com Garcia, "o cotidiano permite tornar a educação significativa, pois conjuga a vivência de situações ao processo do conhecimento, possibilitando o questionamento de práticas sociais e a instrumentalização para o conhecer e o agir" (18). O processo pedagógico utilizado é a problematização (18). Esse se baseia no fato de que, frente às mudanças, o importante não são apenas os conhecimentos, ideias ou comportamentos aprendidos, mas a capacidade do aluno, como participante e agente de transformação social, de detectar os problemas e buscar soluções.

Assim, a capacidade a ser desenvolvida é a de fazer perguntas relevantes, possibilitando o entendimento da situação, e ser capaz de resolvê-las adequadamente. $\mathrm{O}$ processo "ensino-aprendizagem" relacionado a uma determinada realidade se inicia levando os alunos a observar a realidade em si.

\section{Prescrições de fitoterápicos magistrais no ambulatório da ABFIT: fornecimento, distribuição e dispensação}

Os fitoterápicos prescritos para os usuários atendidos no ambulatório da ABFIT são fornecidos e distribuídos pelas farmácias de manipulação parceiras do projeto. Quatro farmácias fazem parte do programa e, a cada semana, uma fica responsável pelo fornecimento das formulações. Além da prática clínica, cabe aos farmacêuticos do ambulatório de fitoterapia receber as formulações, dispensar e orientar o uso correto dos fitoterápicos.

As prescrições devem conter o nome do paciente, nome científico das drogas vegetais, a parte utilizada de cada planta, a padronização (quando houver), a forma farmacêutica, a dose, a posologia e a assinatura do prescritor, bem como o número do registro no conselho de classe $(19,20)$.

A parceria com farmácias magistrais traz o enorme benefício da individualização da formulação de acordo com as necessidades específicas de cada paciente. Além disso, a formulação, que é constituída por mais de uma droga vegetal, também possibilita ampliar a sinergia do fitocomplexo. Assim, a manipulação parte de requisitos próprios: compreender as indicações terapêuticas tradicionais e científicas conhecidas para cada extrato vegetal; entender os parâmetros necessários para a escolha de espécies que melhor se enquadram ao perfil de cada paciente; promover a interface entre a tradição e a ciência.

Segundo o levantamento do Ministério da Saúde, 64,5\% dos municípios não fornecem medicamentos fitoterápicos via farmácia pública de manipulação (13). Isso significa que 
há escassez de recursos públicos no fornecimento de fitoterápicos manipulados na rede pública de saúde. Esse fato corrobora com a necessidade e a importância de farmácias privadas aliadas ao projeto de forma estritamente voluntária.

Em 2017, o Ministério da Saúde atualizou a Relação Nacional de Medicamentos (RENAME), ampliando para 12 o número de fitoterápicos que podem ser disponibilizados pela atenção básica à saúde. No entanto, esses são produtos industrializados com apresentação e formas farmacêuticas padronizadas pela RENAME (21).

Para mudar esse cenário, faz-se necessário o desenvolvimento da PNPIC, que deve ser compreendido como continuidade do processo de implantação do SUS, na medida em que favorece, de forma efetiva, o cumprimento dos princípios e diretrizes que regem o sistema.

Portanto, é notório o diferencial da prescrição dos programas de fitoterapia do SUS em relação à prescrição ambulatorial magistral $(7,8,9,10,11,12)$ uma vez que esta permite adequar as indicações farmacológicas e tradicionais, de forma individualizada, para os usuários atendidos.

\section{Conclusão}

A proposta de prática ambulatorial e de ensino em fitoterapia da ABFIT é considerada um arranjo inovador por se tratar de um atendimento interprofissional que prevê ação conjunta entre as áreas de medicina, nutrição e farmácia. Soma-se a isso o fato de trabalhar com formulações magistrais individualizadas com o diferencial da combinação de elementos médicos tradicionais e conhecimentos técnico-científicos.

Considera-se que o modelo de trabalho e estudo apresentado possa contribuir para o debate sobre a colaboração interprofissional em saúde e sobre a associação de elementos e fatores dos sistemas médicos tradicionais e científicos para o tratamento com fitoterapia.

Espera-se, ainda, que tais ações possam servir de modelo para elaboração de novos projetos ambulatoriais e educacionais em saúde e fitoterapia. Acredita-se que este artigo não esgota o tema, mas o renova e o coloca em discussão.

\section{Referências}

1. World Health Organization (WHO). Legal status of traditional medicine and complementary/alternative medicine. Geneva: World Health Organization; 2001.

2. Organización Mundial de la Salud (OMS). Consejo Ejecutivo. Medicina tradicional y asistencia sanitaria moderna. Foro mundial de la salud. Revista Internacional de Desarrollo Sanitario; 12(1):120. 1991.

3. Organización Mundial de la Salud. Estrategia de la OMS sobre medicina tradicional 2002-2005. Geneva: Organización Mundial de la Salud; 2002.

4. Robinson MM, Zhang X. The world medicines situation 2011: traditional medicines: global situation issues and challenges. $3^{\circ}$ ed. Geneva: WHO, 2011.

5. Matos FJA. Farmácias vivas: sistema de utilização de plantas medicinais projetado para pequenas comunidades. Fortaleza: EUFC; 1998.

6. Sacramento HT. O programa de fitoterapia do município de Vitória-ES. Divulgação em Saúde para Debate 2004; 30:59-65.

7. Graça C. Treze anos de fitoterapia em Curitiba. Divulgação em Saúde para Debate 2004; 30:36-41.

8. Reis MCP, Leda PHO, Pereira MTCL, Tunala EAM. Experiência na implantação do programa de fitoterapia do município do Rio de Janeiro. Divulgação em Saúde para Debate 2004; 30:42-49.

9. Pires AM, Borella JC, Raya LC. Prática alternativa de saúde na atenção básica da rede SUS de Ribeirão Preto-SP. Divulgação em Saúde para Debate 2004; 30:56-58.

10. Carneiro SMC, Pontes LML, Gomes Filho VAF, Guimarães MA. Da planta ao medicamento: experiência da utilização da fitoterapia na atenção primária à saúde no município de Itapioca-CE. 
Divulgação em Saúde para Debate 2004; 30:50-65.

11. Teixeira JBP. Memória institucional da fitoterapia em Juiz de Fora [dissertação de mestrado]. Rio de Janeiro: Instituto de Medicina Social da UERJ; 2003.

12. Nagai SC, Queirós MS. Medicina complementar e alternativa na rede básica de serviços de saúde: uma aproximação qualitativa. Ciênc. Saúde Coletiva 2001; 16(3):1793-800.

13. Brasil. Ministério da Saúde. Secretaria de Atenção à Saúde. Departamento de Atenção Básica. Política Nacional de Práticas Integrativas e Complementares no SUS - PNPIC-SUS / Ministério da Saúde, Secretaria de Atenção à Saúde, Departamento de Atenção Básica. - 2.ed. Brasília: Ministério da Saúde, 2015.

14. Arruda LS, Moreira COF. Colaboração Interprofissional: um estudo de caso do Núcleo de Atenção à saúde do idoso NAI/UERJ. Rio de Janeiro. [Dissertação de Mestrado- ENSP/FIOCRUZ]. 2016.

15. Saad GL, Leda PH, Sá IM, Seixlack AC. Fitoterapia contemporânea: tradição e ciência na prática clínica. $2^{\text {a }}$ ed. Rio de Janeiro: Guanabara Koogan, 2016.

16. Agência Nacional de Vigilância Sanitária - ANVISA. Instrução normativa no 2, de 13 de maio 2014 Publica a "Lista de medicamentos fitoterápicos de registro simplificado" e a "Lista de produtos tradicionais fitoterápicos de registro simplificado". 2014.

17. Who Study Group On Multiprofessional Education of Health Personel: The Team Approach. Learning together to work together for health. Geneva: World Health Organization; 1988.

18. Garcia MAA. Knowledge, action and education: teaching and learning at healthcare centers. Interface 2001; 5(8):89-100.

19. Conselho Federal de Nutricionistas. RESOLUÇÃO CFN No 556, DE 11 DE ABRIL DE 2015. Altera as Resoluções $n^{\circ} 416$, de 2008, e no 525 , de 2013, e acrescenta disposições à regulamentação da prática da Fitoterapia para o nutricionista como complemento da prescrição dietética. 2015. p. 1-3.

20. Conselho Federal de Farmácia. RESOLUÇÃO No 586 DE 29 DE AGOSTO DE 2013. Ementa: Regula a prescrição farmacêutica e dá outras providências. 2013. p. 1-19.

21. Brasil. Ministério da Saúde. Secretaria de Ciência, Tecnologia e Insumos Estratégicos. Departamento de Assistência Farmacêutica e Insumos Estratégicos. Relação Nacional de Medicamentos Essenciais: RENAME 2017 / Ministério da Saúde, Secretaria de Ciência, Tecnologia e Insumos Estratégicos, Departamento de Assistência Farmacêutica e Insumos Estratégicos - Brasília: Ministério da Saúde, 2017. p. 210. 